Commun. Korean Math. Soc. 29 (2014), No. 2, pp. 331-343

http://dx.doi.org/10.4134/CKMS.2014.29.2.331

\title{
ON A SEMI-SYMMETRIC METRIC CONNECTION IN AN $(\varepsilon)$-KENMOTSU MANIFOLD
}

\author{
Ram Nawal Singh, Shravan Kumar Pandey, Giteshwari Pandey, \\ AND KIRAN TIWARI
}

\begin{abstract}
The object of the present paper is to study a semi-symmetric metric connection in an $(\varepsilon)$-Kenmotsu manifold. In this paper, we study a semi-symmetric metric connection in an $(\varepsilon)$-Kenmotsu manifold whose projective curvature tensor satisfies certain curvature conditions.
\end{abstract}

\section{Introduction}

The idea of a semi-symmetric linear connection on a differentiable manifold was first introduced by Friedmann and Schouten [11] in 1924. Hayden [12] introduced a semi-symmetric metric connection on a Riemannian manifold. Yano [21] proved the theorem: A Riemannian manifold admits a semi-symmetric metric connection whose curvature tensor vanishes if and only if Riemannian manifold is conformally flat. Semi-symmetric metric connections on a Riemannian manifold have been studied by Amur and Pujar [1], Pravanovic [15], Binh [4], De ([6], [7]), De and Biswas [8], Sharfuddin and Hussain [16], Pathak and De [14], Jun, De and Pathak [13], Barman and De [2], Chaubey and Ojha [5], Singh and Pandey [17], Singh, Pandey and Pandey ([18], [19]) and many others.

Duggal and Sharma [10] studied a semi-symmetric metric connection in a semi-Riemannian manifold. They studied some properties of the Ricci tensor, affine conformal motions, geodesics and group manifolds with respect to the semi-symmetric metric connection. On the other hand, the study of manifolds with indefinite metrics is of interest from the standpoint of physics and relativity. Manifolds with indefinite metrics have been studied by several authors. In 1993, Bejancu and Duggal [3] introduced the concept of $(\varepsilon)$-Sasakian manifolds and Xufeng and Xiaoli [20] established that these manifolds are real hypersurfaces of indefinite Kahlerian manifolds. Recently De and Sarkar [9] introduced

Received January 8, 2014.

2010 Mathematics Subject Classification. 53C15, 53C25.

Key words and phrases. $(\varepsilon)$-Kenmotsu manifold, semi-symmetric metric connection, quasi-projectively flat $(\varepsilon)$-Kenmotsu manifold, $\phi$-projectively flat $(\varepsilon)$-Kenmotsu manifold, space like, time like. 
$(\varepsilon)$-Kenmotsu manifolds and studied conformally flat, Weyl semisymmetric, $\phi$-recurrent $(\varepsilon)$-Kenmotsu manifolds.

In the present paper, we establish the relation between Levi-Civita connection and semi-symmetric metric connection in an $(\varepsilon)$-Kenmotsu manifold and obtained relation between curvature tensors of Levi-Civita connection and semi-symmetric metric connection. Also some curvature properties of projective curvature tensor with respect to semi-symmetric metric connection in an $(\varepsilon)$-Kenmotsu manifold have been studied.

\section{Preliminaries}

An n-dimensional smooth manifold $\left(M^{n}, g\right)$ is called an $(\varepsilon)$-almost contact metric manifold if

$$
\begin{gathered}
\phi^{2} X=-X+\eta(X) \xi, \\
\eta(\xi)=1, \\
g(\xi, \xi)=\varepsilon, \\
\eta(X)=\varepsilon g(X, \xi), \\
g(\phi X, \phi Y)=g(X, Y)-\varepsilon \eta(X) \eta(Y),
\end{gathered}
$$

where $\varepsilon$ is 1 or -1 according as $\xi$ is space-like or time-like and rank $\phi$ is $n-1$.

It is important to mention that in the above definition $\xi$ is never a light-like vector field.

If

$$
d \eta(X, Y)=g(X, \phi Y)
$$

for every $X, Y \in T M^{n}$, then we say that $M^{n}$ is an $(\varepsilon)$-contact metric manifold. Also,

$$
\phi \xi=0, \quad \eta \circ \phi=0 .
$$

If an $(\varepsilon)$-contact metric manifold satisfies

$$
\left(\nabla_{X} \phi\right)(Y)=-g(X, \phi Y) \xi-\varepsilon \eta(Y) \phi X,
$$

where $\nabla$ denotes the Riemannian connection of $g$, then $M^{n}$ is called an $(\varepsilon)$ Kenmotsu manifold [9].

An $(\varepsilon)$-almost contact metric manifold is an $(\varepsilon)$-Kenmotsu manifold [9] if and only if

$$
\nabla_{X} \xi=\varepsilon(X-\eta(X) \xi) .
$$

In an $(\varepsilon)$-Kenmotsu manifold, the following relations hold [9]

$$
\begin{gathered}
\left(\nabla_{X} \eta\right)(Y)=g(X, Y)-\varepsilon \eta(X) \eta(Y), \\
R(X, Y) \xi=\eta(X) Y-\eta(Y) X,
\end{gathered}
$$




$$
\begin{gathered}
R(\xi, X) Y=\eta(Y) X-\varepsilon g(X, Y) \xi, \\
R(X, Y) \phi Z=\phi R(X, Y) Z+\varepsilon\{g(Y, Z) \phi X-g(X, Z) \phi Y \\
+g(X, \phi Z) Y-g(Y, \phi Z) X\}, \\
\eta(R(X, Y) Z)=\varepsilon[g(X, Z) \eta(Y)-g(Y, Z) \eta(X)], \\
S(X, \xi)=-(n-1) \eta(X), \\
S(\phi X, \phi Y)=S(X, Y)+\varepsilon(n-1) \eta(X) \eta(Y) .
\end{gathered}
$$

Definition 2.1. An $(\varepsilon)$-Kenmotsu manifold $M^{n}$ is said to be $\eta$-Einstein if its Ricci tensor $S$ is of the form

$$
S(X, Y)=a g(X, Y)+b \eta(X) \eta(Y),
$$

where $a$ and $b$ are scalar functions of $\varepsilon$.

In [9], De and Sarkar have given an example of $(\varepsilon)$-Kenmotsu manifold.

Example 1. We consider the three dimensional manifold $M^{3}=\{(x, y, z) \in$ $\left.\mathbb{R}^{3}, z \neq 0\right\}$, where $(x, y, z)$ are the standard coordinates in $\mathbb{R}^{3}$. The vector fields

$$
e_{1}=z \frac{\partial}{\partial x}, e_{2}=z \frac{\partial}{\partial y}, e_{3}=-z \frac{\partial}{\partial z} .
$$

are linearly independent at each point of the manifold. Let $g$ be the Riemannian metric defined by

$$
\begin{aligned}
& g\left(e_{1}, e_{3}\right)=g\left(e_{2}, e_{3}\right)=g\left(e_{2}, e_{2}\right)=0, \\
& g\left(e_{1}, e_{1}\right)=g\left(e_{2}, e_{2}\right)=g\left(e_{3}, e_{3}\right)=\varepsilon,
\end{aligned}
$$

where $\varepsilon= \pm 1$. Let $\eta$ be the 1 -form defined by $\eta(Z)=\varepsilon g\left(Z, e_{3}\right)$ for any $Z \in T M^{n}$.

Let $\phi$ be the $(1,1)$-tensor field defined by

$$
\phi\left(e_{1}\right)=-e_{2}, \phi\left(e_{2}\right)=e_{1}, \phi\left(e_{3}\right)=0 .
$$

Then using the linearity of $\phi$ and $g$, we have

$$
\begin{gathered}
\eta\left(e_{3}\right)=1, \phi^{2} Z=-Z+\eta(Z) e_{3}, \\
g(\phi Z, \phi W)=g(Z, W)-\eta(Z) \eta(W)
\end{gathered}
$$

for any $Z, W \in T M^{n}$.

Let $\nabla$ be the Levi-Civita connection with respect to metric $g$. Then, we have

$$
\left[e_{1}, e_{2}\right]=0,\left[e_{1}, e_{3}\right]=\varepsilon e_{1},\left[e_{2}, e_{3}\right]=\varepsilon e_{2} .
$$

The Riemannian connection $\nabla$ of the metric $g$ is given by

$$
\begin{aligned}
2 g\left(\nabla_{X} Y, Z\right)= & X g(Y, Z)+Y g(Z, X)-Z g(X, Y)-g(X,[(Y, Z])-g(Y,[X, Z]) \\
& +g(Z,[X, Y]),
\end{aligned}
$$


which is known as Koszul's formula. Then Koszul's formula yields

$$
\begin{aligned}
& \nabla_{e_{1}} e_{3}=\varepsilon e_{1}, \nabla_{e_{2}} e_{3}=\varepsilon e_{2}, \nabla_{e_{3}} e_{3}=0, \\
& \nabla_{e_{1}} e_{2}=0, \nabla_{e_{2}} e_{2}=-\varepsilon e_{3}, \nabla_{e_{3}} e_{2}=0, \\
& \nabla_{e_{1}} e_{1}=-\varepsilon e_{3}, \nabla_{e_{2}} e_{1}=0, \nabla_{e_{3}} e_{1}=0 .
\end{aligned}
$$

These results shows that the manifold satisfies

$$
\nabla_{X} \xi=\varepsilon(X-\eta(X) \xi)
$$

for $\xi=e_{3}$. Hence the manifold under consideration is an $(\varepsilon)$-Kenmotsu manifold of dimension three.

\section{Semi-symmetric metric connections}

A linear connection $\bar{\nabla}$ in an almost contact metric manifold $M^{n}$ is said to be semi-symmetric connection [16], if its torsion tensor

$$
\bar{T}(X, Y)=\bar{\nabla}_{X} Y-\bar{\nabla}_{Y} X-[X, Y]
$$

satisfies

$$
\bar{T}(X, Y)=\eta(Y) X-\eta(X) Y .
$$

Further, a semi-symmetric connection is called a semi-symmetric metric connection [21] if

$$
\left(\bar{\nabla}_{X} g\right)(Y, Z)=0 .
$$

Let $M^{n}$ be an n-dimensional $(\varepsilon)$-Kenmotsu manifold and $\nabla$ be the Levi-Civita connection on $M^{n}$. The relation between the semi-symmetric metric connection $\bar{\nabla}$ and the Levi-Civita connection $\nabla$ on $\left(M^{n}, \mathrm{~g}\right)$ is given by

$$
\bar{\nabla}_{X} Y=\nabla_{X} Y+\eta(Y) X-g(X, Y) \xi .
$$

Let $\bar{R}$ be the curvature tensor of semi-symmetric metric connection $\bar{\nabla}$ given by

$$
\bar{R}(X, Y) Z=\bar{\nabla}_{X} \bar{\nabla}_{Y} Z-\bar{\nabla}_{Y} \bar{\nabla}_{X} Z-\bar{\nabla}_{[X, Y]} Z,
$$

which on using equation (3.4) takes the form

$$
\begin{aligned}
\bar{R}(X, Y) Z= & R(X, Y) Z+\left[\left(\nabla_{X} \eta\right)(Z) Y-\left(\nabla_{Y} \eta\right)(Z) X\right] \\
& +\left[g(X, Z)\left(\nabla_{Y} \xi\right)-g(Y, Z)\left(\nabla_{X} \xi\right)\right] \\
& +\eta(Z)[\eta(Y) X-\eta(X) Y]+\eta(\xi)[g(X, Z) Y \\
& -g(Y, Z) X]+[g(Y, Z) g(X, \xi)-g(X, Z) g(Y, \xi)] \xi,
\end{aligned}
$$

where

$$
R(X, Y) Z=\nabla_{X} \nabla_{Y} Z-\nabla_{Y} \nabla_{X} Z-\nabla_{[X, Y]} Z
$$

is the Riemannian curvature tensor of connection $\nabla$. By virtue of equations (2.2), (2.4), (2.9) and (2.10), equation (3.6) reduces to

$$
\begin{aligned}
\bar{R}(X, Y) Z= & R(X, Y) Z+(2+\varepsilon)[g(X, Z) Y-g(Y, Z) X] \\
& +(1+\varepsilon)[g(Y, Z) \eta(X)-g(X, Z) \eta(Y)] \xi
\end{aligned}
$$




$$
+(1+\varepsilon) \eta(Z)[\eta(Y) X-\eta(X) Y]
$$

which is the relation between curvature tensors of connection $\bar{\nabla}$ and $\nabla$. From equation (3.8), we have

$$
\begin{aligned}
& ' \bar{R}(X, Y, Z, U) \\
= & ' R(X, Y, Z, U)+(2+\varepsilon)[g(X, Z) g(Y, U)-g(Y, Z) g(X, U)] \\
& +(1+\varepsilon)[g(Y, Z) \eta(X)-g(X, Z) \eta(Y)] \eta(U) \\
& +(1+\varepsilon)[g(X, U) \eta(Y)-g(Y, U) \eta(X)] \eta(Z),
\end{aligned}
$$

where ' $\bar{R}(X, Y, Z, U)=g(\bar{R}(X, Y) Z, U)$ and ' $R(X, Y, Z, U)=g(R(X, Y) Z, U)$. Putting $X=U=e_{i}$ in the above equation and taking summation over $i, 1 \leq$ $i \leq n$, we get

$$
\begin{aligned}
\bar{S}(Y, Z)= & S(Y, Z)+[(\varepsilon+2)(\varepsilon-n)+2] g(Y, Z) \\
& +(1+\varepsilon)(n-2 \varepsilon) \eta(Y) \eta(Z),
\end{aligned}
$$

where $\bar{S}$ and $S$ are the Ricci tensors of connection $\bar{\nabla}$ and $\nabla$, respectively in $M^{n}$. Contracting the above equation, we get

$$
\bar{r}=r+\eta((\varepsilon+2)(\varepsilon-n)+2)+\varepsilon(1+\varepsilon)(n-2 \varepsilon),
$$

where $\bar{r}$ and $r$ are the scalar curvatures of the connection $\bar{\nabla}$ and $\nabla$, respectively.

Writing two more equations by the cyclic permutations of $X, Y$ and $Z$, we get

$$
\begin{aligned}
\bar{R}(Y, Z) X= & R(Y, Z) X+(2+\varepsilon)[g(Y, X) Z-g(Z, X) Y] \\
& +(1+\varepsilon)[g(Z, X) \eta(Y)-g(Y, X) \eta(Z)] \xi \\
& +(1+\varepsilon)[\eta(Z) Y-\eta(Y) Z] \eta(X)
\end{aligned}
$$

and

$$
\begin{aligned}
\bar{R}(Z, X) Y= & R(Z, X) Y+(2+\varepsilon)[g(Z, Y) X-g(X, Y) Z] \\
& +(1+\varepsilon)[g(X, Y) \eta(Z)-g(Z, Y) \eta(X)] \xi \\
& +(1+\varepsilon)[\eta(X) Z-\eta(Z) Y] \eta(Y) .
\end{aligned}
$$

Adding equations (3.8), (3.12) and (3.13) and using the Bianchi's first identity, we get

$$
\bar{R}(X, Y) Z+\bar{R}(Y, Z) X+\bar{R}(Z, X) Y=0 .
$$

Thus, we can state as follows:

Theorem 3.1. An $(\varepsilon)$-Kenmotsu manifold $M^{n}$ with semi-symmetric metric connection satisfies the equation (3.14).

Now interchanging $X$ and $Y$ in the equation (3.9), we get

$$
\begin{aligned}
& ' \bar{R}(Y, X, Z, U) \\
= & ' R(Y, X, Z, U)+(2+\varepsilon)[g(Y, Z) g(X, U)-g(X, Z) g(Y, U)]
\end{aligned}
$$




$$
\begin{aligned}
& +(1+\varepsilon)[g(X, Z) \eta(Y)-g(Y, Z) \eta(X)] \eta(U) \\
& +(1+\varepsilon)[g(Y, U) \eta(X)-g(X, U) \eta(Y)] \eta(Z) .
\end{aligned}
$$

Adding equations (3.9) and (3.15) with the fact that

$$
{ }^{\prime} R(X, Y, Z, U)+{ }^{\prime} R(Y, X, Z, U)=0,
$$

we get

$$
{ }^{\prime} \bar{R}(X, Y, Z, U)+{ }^{\prime} \bar{R}(Y, X, Z, U)=0 .
$$

Again interchanging $Z$ and $U$ in the equation (3.9), we get

$$
\begin{aligned}
& ' \bar{R}(X, Y, U, Z) \\
= & ' R(X, Y, U, Z)+(2+\varepsilon)[g(X, U) g(Y, Z)-g(Y, U) g(X, Z)] \\
& +(1+\varepsilon)[g(Y, U) \eta(X)-g(X, U) \eta(Y)] \eta(Z) \\
& +(1+\varepsilon)[g(X, Z) \eta(Y)-g(Y, Z) \eta(X)] \eta(U) .
\end{aligned}
$$

Now adding equations (3.9) and (3.17) with the fact that

$$
{ }^{\prime} \bar{R}(X, Y, Z, U)+{ }^{\prime} \bar{R}(X, Y, U, Z)=0,
$$

we get

$$
{ }^{\prime} \bar{R}(X, Y, Z, U)+{ }^{\prime} \bar{R}(X, Y, U, Z)=0 .
$$

Again interchanging pair of slots in the equation (3.9), we get

$$
\begin{aligned}
& { }^{\prime} \\
= & { }^{\prime}(Z, U, X, Y) \\
& +(1+\varepsilon)[g(U, X) \eta(Z)-g(Z, X) \eta(U)] \eta(Y) \\
& +(1+\varepsilon)[g(Z, Y) \eta(U)-g(U, Y) \eta(Z)] \eta(X) .
\end{aligned}
$$

Now subtracting the equation (3.19) from the equation (3.9) with the fact that

$$
{ }^{\prime} R(X, Y, Z, U)-{ }^{\prime} R(Z, U, X, Y)=0,
$$

we get

$$
{ }^{\prime} \bar{R}(X, Y, Z, U)-^{\prime} \bar{R}(Z, U, X, Y)=0 .
$$

Thus, in view of equations (3.16), (3.18) and (3.20) we can state as follows:

Theorem 3.2. The curvature tensor of type $(0,4)$ of a semi-symmetric metric connection in an $(\varepsilon)$-Kenmotsu manifold is

(i) Skew-symmetric in first two slots,

(ii) Skew-symmetric in last two slots,

(iii) Symmetric in pair of slots.

Now, let $\bar{R}(X, Y) Z=0$, which by virtue of the equation (3.8) yields

$$
\begin{aligned}
R(X, Y) Z= & (2+\varepsilon)[g(Y, Z) X-g(X, Z) Y]+(1+\varepsilon)[g(X, Z) \eta(Y) \\
& -g(Y, Z) \eta(X] \xi+(1+\varepsilon)[\eta(X) Y-\eta(Y) X] \eta(Z) .
\end{aligned}
$$


Taking the inner product of the above equation with $\xi$, we get

$$
\varepsilon \eta(R(X, Y) Z)=\varepsilon[g(Y, Z) \eta(X)-g(X, Z) \eta(Y)],
$$

which by virtue of equation (2.4) gives

$$
R(X, Y) Z=[g(Y, Z) X-g(X, Z) Y]
$$

The above equation can be written as

$$
{ }^{\prime} R(X, Y, Z, U)=g(Y, Z) g(X, U)-g(X, Z) g(Y, U) .
$$

Thus, we can state as follows:

Theorem 3.3. If the curvature tensor of a semi-symmetric metric connection in an $(\varepsilon)$-Kenmotsu manifold $M^{n}$ vanishes, then the manifold is of constant curvature.

Now suppose Ricci tensor of a semi-symmetric metric connection in $M^{n}$ vanishes, i.e., $\bar{S}(Y, Z)=0$, then from the equation (3.10) we have

$$
S(Y, Z)=-[(\varepsilon+2)(\varepsilon-n)+2] g(Y, Z)-(1+\varepsilon)(n-2 \varepsilon) \eta(Y) \eta(Z),
$$

which is

$$
S(Y, Z)=a g(Y, Z)+b \eta(Y) \eta(Z),
$$

where $a=-[(\varepsilon+2)(\varepsilon-n)+2]$ and $b=-[(1+\varepsilon)(n-2 \varepsilon)]$. Thus, from the above discussion, we can state the following:

Theorem 3.4. If the Ricci tensor of the semi-symmetric metric connection $\bar{\nabla}$ in an $(\varepsilon)$-Kenmotsu manifold vanishes, then the manifold $M^{n}$ is $\eta$-Einstein manifold.

\section{Quasi-projectively flat $(\varepsilon)$-Kenmotsu manifold with respect to semi-symmetric metric connection}

The projective curvature tensor is an important tensor from the differential geometric point of view. Let $M^{n}$ be an $n$-dimensional Riemannian manifold. If there exists a one to one correspondence between each coordinate neighbourhood of $M^{n}$ and a domain in Euclidian space such that any geodesic of the Riemannian manifold corresponds to a straight line in the Euclidean space, then $M^{n}$ is said to be locally projectively flat [2]. The projective curvature tensor $\bar{P}$ with respect to semi-symmetric metric connection is defined by

$$
\bar{P}(X, Y) Z=\bar{R}(X, Y) Z-\frac{1}{(n-1)}[\bar{S}(Y, Z) X-\bar{S}(X, Z) Y] .
$$

Definition 4.1. An $(\varepsilon)$-Kenmotsu manifold $M^{n}$ is said to be quasi-projectively flat with respect to semi-symmetric metric connection, if

$$
g(\bar{P}(\phi X, Y) Z, \phi W)=0,
$$

where $\bar{P}$ is the projective curvature tensor with respect to semi-symmetric metric connection. 
In view of the equation (4.1), we have

$$
\begin{aligned}
& g(\bar{P}(X, Y) Z, W) \\
= & g(\bar{R}(X, Y) Z, W)-\frac{1}{(n-1)}[\bar{S}(Y, Z) g(X, W)-\bar{S}(X, Z) g(Y, W)] .
\end{aligned}
$$

Putting $X=\phi X$ and $W=\phi W$ in the above equation, we get

$$
\begin{aligned}
& g(\bar{P}(\phi X, Y) Z, \phi W) \\
= & g(\bar{R}(\phi X, Y) Z, \phi W)-\frac{1}{(n-1)}[\bar{S}(Y, Z) g(\phi X, \phi W)-\bar{S}(\phi X, Z) g(Y, \phi W)] .
\end{aligned}
$$

Now assume that $M^{n}$ is quasi-projectively flat with respect to semi-symmetric metric connection. Then by virtue of equations (4.2) and (4.4), we have

(4.5) $g(\bar{R}(\phi X, Y) Z, \phi W)=\frac{1}{(n-1)}[\bar{S}(Y, Z) g(\phi X, \phi W)-\bar{S}(\phi X, Z) g(Y, \phi W)]$.

Using equations (3.8) and (3.10) in the above equations, we get

$$
\begin{aligned}
& g(R(\phi X, Y) Z, \phi W) \\
= & \frac{1}{(n-1)}[S(Y, Z) g(\phi X, \phi W)-S(\phi X, Z) g(Y, \phi W)] \\
& +\left(\frac{(\varepsilon+2)(\varepsilon-1)+2}{(n-1)}\right)[g(Y, Z) g(\phi X, \phi W)-g(\phi X, Z) g(Y, \phi W)] \\
& +\left(\frac{(1+\varepsilon)(1-2 \varepsilon)}{(n-1)}\right) g(\phi X, \phi W) \eta(Y) \eta(Z) .
\end{aligned}
$$

Let $\left\{e_{1}, e_{2}, \ldots, e_{n-1}, \xi\right\}$ be a local orthonormal basis of vector fields in $M^{n}$. Then $\left\{\phi e_{1}, \phi e_{2}, \ldots, \phi e_{n-1}, \xi\right\}$ is also a local orthonormal basis of $M^{n}$. Putting $X=W=e_{i}$ in the equation (4.6) and taking summation over $i, 1 \leq i \leq n-1$, we get

$$
\begin{aligned}
& \sum_{i=1}^{n-1} g\left(R\left(\phi e_{i}, Y\right) Z, \phi e_{i}\right) \\
= & \frac{1}{(n-1)} \sum_{i=1}^{n-1}\left[S(Y, Z) g\left(\phi e_{i}, \phi e_{i}\right)-S\left(\phi e_{i}, Z\right) g\left(Y, \phi e_{i}\right)\right] \\
& +\left(\frac{(\varepsilon+2)(\varepsilon-1)+2}{(n-1)}\right) \sum_{i=1}^{n-1}\left[g(Y, Z) g\left(\phi e_{i}, \phi e_{i}\right)-g\left(\phi e_{i}, Z\right) g\left(Y, \phi e_{i}\right)\right] \\
& +\frac{(1+\varepsilon)(1-2 \varepsilon)}{(n-1)} \sum_{i=1}^{n-1} g\left(\phi e_{i}, \phi e_{i}\right) \eta(Y) \eta(Z) .
\end{aligned}
$$


Also,

$$
\begin{gathered}
\sum_{i=1}^{n=1} g\left(R\left(\phi e_{i}, Y\right) Z, \phi e_{i}\right)=S(Y, Z)+g(Y, Z), \\
\sum_{i=1}^{n=1} g\left(\phi e_{i}, Y\right) S\left(\phi e_{i}, Z\right)=S(Y, Z), \\
\sum_{i=1}^{n=1} g\left(\phi e_{i}, \phi e_{i}\right)=n-1,
\end{gathered}
$$

and

$$
\sum_{i=1}^{n-1} g\left(\phi e_{i}, Z\right) g\left(Y, \phi e_{i}\right)=g(Y, Z) .
$$

Hence, by virtue of equations (4.8), (4.9), (4.10) and (4.11) the equation (4.7) takes the form

$$
S(Y, Z)=[(n-2) \varepsilon-1](g(Y, Z)+(-1-\varepsilon)(n-1) \eta(Y) \eta(Z),
$$

which is

$$
S(Y, Z)=a g(Y, Z)+b \eta(Y) \eta(Z),
$$

where $a=(n-2) \varepsilon-1$ and $b=(-1-\varepsilon)(n-1)$. This shows that $M^{n}$ is an $\eta$-Einstein manifold. Thus, we can state as follows.

Theorem 4.1. A quasi-projectively flat ( $(\varepsilon)$-Kenmotsu manifold with respect to a semi-symmetric metric connection is an $\eta$-Einstein manifold.

\section{5. $\phi$-projectively flat $(\varepsilon)$-Kenmotsu manifold with respect to a semi-symmetric metric connection}

Definition 5.1. An $(\varepsilon)$-Kenmotsu manifold with respect to a semi-symmetric metric connection is said to be $\phi$-projectively flat if

$$
\phi^{2}(\bar{P}(\phi X, \phi Y) \phi Z)=0,
$$

where $\bar{P}$ is the projective curvature tensor of the manifold $M^{n}$ with respect to semi-symmetric metric connection.

Let $M^{n}$ be a $\phi$-projectively flat $(\varepsilon)$-Kenmotsu manifold with respect to semisymmetric metric connection. It is easy to see that $\phi^{2}(\bar{P}(\phi X, \phi Y) \phi Z)=0$ holds if and only if

$$
g(\bar{P}(\phi X, \phi Y) \phi Z, \phi W)=0
$$

for any $X, Y, Z, W \in T M^{n}$.

Putting $Y=\phi Y$ and $Z=\phi Z$ in the equation (4.4), we get

$$
\begin{aligned}
& g(\bar{P}(\phi X, \phi Y) \phi Z, \phi W) \\
= & g(\bar{R}(\phi X, \phi Y) \phi Z, \phi W)
\end{aligned}
$$




$$
-\frac{1}{(n-1)}[\bar{S}(\phi Y, \phi Z) g(\phi X, \phi W)-\bar{S}(\phi X, \phi Z) g(\phi Y, \phi W)] .
$$

Using the equation (5.3) in the equation (5.2), we obtain

$$
\begin{aligned}
g(\bar{R}(\phi X, \phi Y) \phi Z, \phi W)= & \frac{1}{(n-1)}[\bar{S}(\phi Y, \phi Z) g(\phi X, \phi W) \\
& -\bar{S}(\phi X, \phi Z) g(\phi Y, \phi W)],
\end{aligned}
$$

which on using equations (3.8) and (3.10) gives

$$
\begin{aligned}
& g(R(\phi X, \phi Y) \phi Z, \phi W) \\
= & \frac{1}{(n-1)}[S(\phi Y, \phi Z) g(\phi X, \phi W)-\bar{S}(\phi X, \phi Z) g(\phi Y, \phi W)] \\
& +\frac{(1+\varepsilon)}{(n-1)}[g(\phi Y, \phi Z) g(\phi X, \phi W)-g(\phi X, \phi Z) g(\phi Y, \phi W)] .
\end{aligned}
$$

Let $\left\{e_{1}, e_{2}, \ldots, e_{n-1}, \xi\right\}$ be a local orthonormal basis of vector fields in $M^{n}$. We Use the fact that $\left\{\phi e_{1}, \phi e_{2}, \ldots, \phi e_{n-1}, \xi\right\}$ is also orthonormal basis. Putting $X=W=e_{i}$ in equation (5.5) and summing over $i$, we get

$$
\begin{aligned}
& \sum_{i=1}^{n-1} g\left(R\left(\phi e_{i}, \phi Y\right) \phi Z, \phi e_{i}\right) \\
= & \frac{1}{(n-1)} \sum_{i=1}^{n-1}\left[S(\phi Y, \phi Z) g\left(\phi e_{i}, \phi e_{i}\right)-S\left(\phi e_{i}, \phi Z\right) g\left(\phi Y, \phi e_{i}\right)\right] \\
& +\frac{(1+\varepsilon)}{(n-1)} \sum_{i=1}^{n-1}\left[g(\phi Y, \phi Z) g\left(\phi e_{i}, \phi e_{i}\right)-g\left(\phi e_{i}, \phi Z\right) g\left(\phi Y, \phi e_{i}\right)\right],
\end{aligned}
$$

which by virtue of equations (4.8), (4.9), (4.10) and (4.11) takes the form

$$
S(\phi Y, \phi Z)=[(n-2) \varepsilon-1] g(\phi Y . \phi Z) .
$$

Using equations (2.5) and (2.16) in the above equations, we get

$$
S(Y, Z)=((n-2) \varepsilon-1) g(Y, Z)+(2-n)(1+\varepsilon) \eta(Y) \eta(Z),
$$

which is of the form

$$
S(Y, Z)=a g(Y, Z)+b \eta(Y) \eta(Z),
$$

where $a=(n-2) \varepsilon-1$ and $b=(2-n)(1+\varepsilon)$. This shows that $M^{n}$ is an $\eta$-Einstein manifold.

Thus, we can state as follows.

Theorem 5.1. $\phi$-Projectively flat $(\varepsilon)$-Kenmotsu manifold with respect to a semi-symmetric metric connection is an $\eta$-Einstein manifold. 


\section{6. $(\varepsilon)$-Kenmotsu manifold with respect to a semi-symmetric metric connection satisfying $\bar{P} \cdot \bar{S}=0$}

Consider $(\varepsilon)$-Kenmotsu manifold with respect to a semi-symmetric metric connection satisfying

$$
(\bar{P}(X, Y) \cdot \bar{S})(Z, U)=0,
$$

where $\bar{S}$ is the Ricci tensor with respect to a semi-symmetric metric connection. Then, we have

$$
\bar{S}(\bar{P}(X, Y) Z, U)+\bar{S}(Z, \bar{P}(X, Y) U)=0 .
$$

Putting $X=\xi$ in the equation (6.2), we have

$$
\bar{S}(\bar{P}(\xi, Y) Z, U)+\bar{S}(Z, \bar{P}(\xi, Y) U)=0 .
$$

In view of the equation (4.1), we have

$$
\bar{P}(\xi, Y) Z=\bar{R}(\xi, Y) Z-\frac{1}{(n-1)}[\bar{S}(Y, Z) \xi-\bar{S}(\xi, Z) Y] .
$$

By virtue of equations (3.8) and (3.10), we have

$$
\bar{R}(\xi, Y) Z=R(\xi, Y) Z+\varepsilon \eta(Z) Y-g(Y, Z) \xi
$$

and

$$
\bar{S}(\xi, Z)=S(\xi, Z)-(n-1) \varepsilon \eta(Z) .
$$

Using equations (6.5) and (6.6) in the equation (6.4), we get

$$
\begin{aligned}
\bar{P}(\xi, Y) Z= & -\left(\frac{\varepsilon-n+2}{n-1}\right) g(Y, Z) \xi-\frac{1}{(n-1)} S(Y, Z) \xi \\
& -\frac{(n-2)(1+\varepsilon)}{(n-1)} \eta(Y) \eta(Z) \xi .
\end{aligned}
$$

Now using the equation (6.7) in the equation (6.3), we get

(6.8) $(\varepsilon-n+2)[g(Y, Z) \eta(U)+g(Y, U) \eta(Z)]+[S(Y, Z) \eta(U)+S(Y, U) \eta(Z)]$

$$
+2(n-2)(1+\varepsilon) \eta(Y) \eta(Z) \eta(U)=0 .
$$

Putting $U=\xi$ in the above equation and using equations (2.2) and (2.15), we get

$$
S(Y, Z)=(n-\varepsilon+2) g(Y, Z)+(1+\varepsilon)(2-n) \eta(Y) \eta(Z),
$$

which is of the form

$$
S(Y, Z)=a g(Y, Z)+b \eta(Y) \eta(Z),
$$

where $a=n-\varepsilon+2$ and $b=(1+\varepsilon)(2-n)$.

This shows that $M^{n}$ is an $\eta$-Einstein manifold. Thus, we can state as follows.

Theorem 6.1. An $(\varepsilon)$-Kenmotsu manifold $M^{n}$ with a semi-symmetric metric connection satisfying $\bar{P} \cdot \bar{S}=0$, is an $\eta$-Einstein manifold. 


\section{References}

[1] K. Amur and S. S. Pujar, On submanifolds of a Riemannian manifold admitting a metric semi-symmetric connection, Tensor (N.S.) 32 (1978), no. 1, 35-38.

[2] A. Barman and U. C. De, Projective curvature tensor of a semi-symmetric metric connection in a Kenmotsu manifold, Int. Electron. J. Geom. 6 (2013), no. 1, 159-169.

[3] A. Bejancu and K. L. Duggal, Real hypersurfaces of indefinite Kahler manifolds, Int. J. Math. Math. Sci. 16 (1993), no. 3, 545-556.

[4] T. Q. Binh, On semi-symmetric connection, Period. Math. Hungar. 21 (1990), no. 2, 101-107.

5] S. K. Chaubey and R. H. Ojha, On a semi-symmetric non-metric connection, Filomat 26 (2012), no. 2, 269-275.

[6] U. C. De, On a type of semi-symmetric connection on a Riemannian manifold, Indian J. Pure Appl. Math. 21 (1990), no. 4, 334-338.

7] - On a type of semi-symmetric metric connection on a Riemannian manifold, An. Stiint. Univ. Al. I. Cuza Iasi Sect. I a Mat. 37 (1991), no. 1, 105-108

[8] U. C. De and S. C. Biswas, On a type of semi-symmetric metric connection on a Riemannian manifold, Pub. Inst. Math. (Beograd) (N.S.) 61(75) (1997), 90-96.

[9] U. C. De and A. Sarkar, On $\varepsilon$-Kenmotsu manifold, Hadronic J. 32 (2009), no. 2, 231242 .

[10] K. L. Duggal and R. Sharma, Semi-symmetric metric connection in a semi-Riemannian manifold, Indian J. Pure Appl. Math. 17 (1986), no. 11, 1276-1283.

[11] A. Friedmann and J. A. Schouten, Uber die geometric der halbsymmetrischen Ubertragung, Math. Zeitschr 21 (1924), 211-223.

[12] H. A. Hayden, Subspaces of space with torsion, Proc. London Math. Soc. 34 (1932), $27-50$.

[13] J. B. Jun, U. C. De, and G. Pathak, On Kenmotsu manifolds, J. Korean Math. Soc. 42 (2005), no. 3, 435-445.

[14] G. Pathak and U. C. De, On a semi-symmetric connection in a Kenmotsu manifold, Bull. Calcutta Math. Soc. 94 (2002), no. 4, 319-324.

[15] M. Prvanovic, On pseudo metric semi-symmetric connections, Pub. De L Institut Math. Nouvelle Series 18 (1975), no. 32, 157-164.

[16] A. Sharfuddin and S. I. Hussain, Semi-symmetric metric connections in almost contact manifolds, Tensor (N.S.) 30 (1976), 133-139.

[17] R. N. Singh and M. K. Pandey, On a type of semi-symmetric metric connection on a Riemannian manifold, Rev. Bull. Calcutta Math. Soc. 16 (2008), no. 2, 179-184.

[18] R. N. Singh, S. K. Pandey, and G. Pandey, Some curvature properties of a semisymmetric metric connection in a Kenmotsu manifold, Rev. Bull. Calcutta Math. Soc. 20 (2012), no. 1, 81-92.

[19] On a semi-symmetric metric connection in an SP-Sasakian manifold, Proc. Natl. Acad. Sci. India. Sect. A 83 (2013), no. 1, 39-47.

[20] X. Xufeng and C. Xiaoli, Two theorems on $(\varepsilon)$-Sasakian manifolds, Internat. J. Math. Math. Sci. 21 (1998), no. 2, 249-254.

[21] K. Yano, On semi-symmetric metric connection, Rev. Roumaine Math. Pure Appl. 15 (1970), 1570-1586.

RAM NAWAL SINGH

Department of Mathematical Sciences

A.P.S. UNIVERSITY

REWA(M.P.), 486003, INDIA

E-mail address: rnsinghmp@rediffmail.com 
Shravan Kumar Pandey

Department of Mathematical Sciences

A.P.S. UNIVERSity

REWA(M.P.), 486003, INDIA

E-mail address: shravan.math@gmail.com

Giteshwari Pandey

Department of Mathematical Sciences

A.P.S. University

REWA(M.P.), 486003, INDIA

E-mail address: math.giteshwari@gmail.com

KIRAN TIWARI

Department of Mathematical Sciences

A.P.S. UNIVERSITY

REWA(M.P.), 486003, IndiA

E-mail address: kirantiwari@yahoo.com 